\title{
First law of black hole in the gravitational electromagnetic system
}

\author{
Jie Jiang, ${ }^{a, b}$ Aofei Sang ${ }^{a, b}$ and Ming Zhang ${ }^{c, 1}$ \\ ${ }^{a}$ College of Education for the Future, Beijing Normal University, \\ Zhuhai, 51908\%, China \\ ${ }^{b}$ Department of Physics, Beijing Normal University, \\ Beijing, 100875, China \\ ${ }^{c}$ Department of Physics, Jiangxi Normal University, \\ Nanchang, 330022, China \\ E-mail: jiejiang@mail.bnu.edu.cn, 202021140021@mail.bnu.edu.cn, \\ mingzhang@jxnu.edu.cn
}

Abstract: After considering the quantum corrections of Einstein-Maxwell theory, the effective theory will contain some higher-curvature terms and nonminimally coupled electromagnetic fields. In this paper, we study the first law of black holes in the gravitational electromagnetic system with the Lagrangian $\mathcal{L}\left(g_{a b}, R_{a b c d}, F_{a b}\right)$. Firstly, we calculate the Noether charge and the variational identity in this theory, and then generically derive the first law of thermodynamics for an asymptotically flat stationary-axisymmetric symmetric black hole without the requirement that the electromagnetic field is smooth on the bifurcation surface. Our results indicate that the first law of black hole thermodynamics might be valid for the Einstein-Maxwell theory with some quantum corrections in the effective region.

KEywords: Black Holes, Classical Theories of Gravity

ARXIV EPRINT: 2108.00766

\footnotetext{
${ }^{1}$ Corresponding author.
} 


\section{Contents}

$\begin{array}{llr}1 & \text { Introduction } & 1\end{array}$

2 Noether charge in the gravitational electromagnetic system 2

3 The first law of the stationary-axisymmetric black holes $\quad 8$

$\begin{array}{lll}4 & \text { Conclusion } & 12\end{array}$

\section{Introduction}

General relativity is the most successful theory to describe the interaction of gravity. It predicts the existence of the black hole, which is a fundamental object in theoretical physics, astronomy, and cosmology. Over the past few decades, many studies of general relativity have shown that black holes can be viewed as a thermodynamic system and satisfy the four laws of thermodynamics [1-3]. By considering the semi-classical quantum effect in curved spacetime, Hawking found that the black hole can be regarded as a blackbody system [4], which provides a natural provided a natural explanation to the laws of black hole thermodynamics. After that, the thermodynamics of black holes has aroused wide interest among researchers, and people believe that it can give us a deeper understanding of gravity.

The most profound laws of black hole mechanics are the first and second laws. With a straightforward derivation, the first law of the Kerr-Newmann black hole shows the relationship between the variations of black hole mass $M$, angular momentum $J$, electric charge $Q$, and areas, i.e.,

$$
\delta M=\frac{\kappa}{8 \pi} \delta A+\Omega_{H} \delta J+\Phi_{H} \delta Q,
$$

in which $\kappa, \Omega_{H}$, and $\Phi_{H}$ are the surface gravity, angular velocity, and electric potential of the event horizon. The original derivation of the first law demands that the perturbation of the spacetime is stationary ("equilibrium state version") [2]. Moreover, their calculation is also based on the Einstein equation. After that, the discussion is extended to the "physical process version", where a stationary black hole is changed by some infinitesimal physical process $[5,6]$. In particular, Iyer and Wald $[6,7]$ show that the above first law of thermodynamic relation is also applicable to any diffeomorphism covariant theories, in which the first law of black holes can be regarded as a straightforward result of the variational identity, and it can be expressed as

$$
\delta M=\frac{\kappa}{2 \pi} \delta S+\Omega_{H} \delta J,
$$


where

$$
S \equiv-2 \pi \int_{\mathcal{B}} \tilde{\boldsymbol{\epsilon}} \frac{\delta \mathcal{L}}{\delta R_{a b c d}} \hat{\boldsymbol{\epsilon}}_{a b} \hat{\boldsymbol{\epsilon}}_{c d}
$$

is the Wald entropy which can be expressed as a Noether charge of theory [6,7], where $\boldsymbol{\epsilon}_{a b}$ is the binormal of the cross-section $\mathcal{B}$ of the event horizon.

It is worth noting that Iyer-Wald's discussion does not consider the cases with the gauge field, and they assume that the asymptotically flat stationary black hole contains a bifurcated Killing horizon and all fields are smooth on the Killing horizon as well as the bifurcation surface [6]. In the gravitational electromagnetic system, because of the gauge covariance of the electromagnetic field, the vector potential is not a real physical quantity in spacetime. Therefore it is not necessary to demand that the vector potential is smooth on the Killing horizon, and Iyer-Wald's results cannot be simply extended to these cases. With this consideration, Gao derived the first law of the asymptotically flat stationary black holes in Einstein-Maxwell and Einstein-Yang-Mills theories without the assumption that the vector potential is smooth on the Killing horizon [8]. Their result shows the same expression as eq. (1.1). Moreover, it has been shown that the first law is satisfied for the Einstein gravity minimally coupled nonlinear electromagnetic field [9]. For the cases with non-minimal couplings, the first law has been explicitly checked for some particular theories [10]. Recently, the discussion is also extended to some cases where the fields have internal gauge freedom based on the extension of Wald's formalism [11-13].

The standard Einstein-Maxwell is a good approximation to describe the gravitational and electromagnetic interactions at a low-energy regime. However, at higher-energy regime, the effective theory should be corrected by adding some higher-order derivative terms to take into account the quantum effects [14-17], including the higher-curvature terms and nonminimally coupled electromagnetic field terms. These corrections will modify the dynamics of gravity as well as the laws of black holes. A natural question is whether the first law of black holes is also satisfied in these effective theories after the quantum corrections are taken into account. Therefore, in this paper, we would like to extend Gao's discussion [8] into a more general gravitational electromagnetic system and derive the first law of black holes without the assumption that the vector potential is smooth at the Killing horizon.

The remainder of this paper is organized as follows. In the next section, we derive the explicit expressions of the Noether charge and variational identity in the gravitational electromagnetic theory with a general Lagrangian $\mathcal{L}\left(g_{a b}, R_{a b c d}, F_{a b}\right)$. In section 3 , after assuming that the metric and electromagnetic strength are smooth near the Killing horizon as well as the bifurcation surface, we derive the first law of the black hole thermodynamics in the gravitational electromagnetic system. Finally, we give a brief conclusion in section 4 .

\section{Noether charge in the gravitational electromagnetic system}

In this section, we first review the Noether current and Noether charge in the diffeomorphism covariant gravitational electromagnetic theory. The Lagrangian $n$-form is given by

$$
\boldsymbol{L}=\boldsymbol{\epsilon} \mathcal{L}\left(g_{a b}, R_{a b c d}, F_{a b}\right),
$$


in which $\boldsymbol{F}=d \boldsymbol{A}$ with the vector potential $\boldsymbol{A}$ is the electromagnetic strength, $R_{a b c d}$ is the Riemann curvature tensor of the Lorentz signature metric $g_{a b}$, and $\mathcal{L}$ is an analytic function of the scalars from the contraction of $R_{a b c d}$ and $F_{a b}$. In the following, we refer to $\left(g_{a b}, \psi\right)$ as $\phi$ collectively. Consider a one-parameter family $\phi(\lambda)$ of the configuration space. The variation of any quantity $\eta(\lambda)$ is defined by

$$
\delta \phi=\left.\frac{d \phi(\lambda)}{d \lambda}\right|_{\lambda=0}
$$

Variation of the Lagrangian $n$-form can be formally divided as

$$
\delta \boldsymbol{L}=\boldsymbol{E}_{\phi} \delta \phi+d \boldsymbol{\Theta}(\phi, \delta \phi),
$$

in which $\boldsymbol{E}_{\phi}=0$ is the equation of motion and $\boldsymbol{\Theta}(\phi, \delta \phi)$ is the symplectic potential of this theory. Next, we are going to calculate the explicit expression of these quantities. From eq. (2.1), we have

$$
\begin{aligned}
\delta \boldsymbol{L} & =\boldsymbol{\epsilon} \delta \mathcal{L}+(\delta \boldsymbol{\epsilon}) \mathcal{L} \\
& =\boldsymbol{\epsilon} \delta \mathcal{L}+\frac{1}{2} \boldsymbol{L} g^{a b} \delta g_{a b} .
\end{aligned}
$$

For the first term of the above equation, we have

$$
\delta \mathcal{L}=A^{a b} \delta g_{a b}+E_{R}^{a b c d} \delta R_{a b c d}+E_{F}^{a b} \delta F_{a b},
$$

in which we have denoted

$$
A^{a b}=\frac{\partial \mathcal{L}}{\partial g_{a b}}, \quad E_{R}^{a b c d}=\frac{\partial \mathcal{L}}{\partial R_{a b c d}}, \quad E_{F}^{a b}=\frac{\partial \mathcal{L}}{\partial F_{a b}} .
$$

For the first term of eq. (2.5), using the relation

$$
A^{a b} \delta g_{a b}=-\frac{\partial \mathcal{L}}{\partial g^{a b}} \delta g^{a b}
$$

we have

$$
A_{a b}=-\frac{\partial \mathcal{L}}{\partial g^{a b}} .
$$

Considering the assumption that $\mathcal{L}$ is a function of the contractions of $R_{a b c d}$ and $F_{a b}$, it is not hard to get

$$
\frac{\partial \mathcal{L}}{\partial g^{a b}}=2\left(E_{R}\right)_{a}{ }^{c d e} R_{b c d e}+\left(E_{F}\right)_{a}{ }^{c} F_{b c} .
$$

After noting that the index of " $a$ " and " $b$ " in the above expression is symmetric, we also have

$$
2\left(E_{R}\right)_{[a}{ }^{c d e} R_{b] c d e}+\left(E_{F}\right)_{[a}{ }^{c} F_{b] c}=0 .
$$

For the second term of eq. (2.5), we have

$$
\begin{aligned}
E_{R}^{a b c d} \delta R_{a b c d} & =E_{R}^{a b c d} R_{a b c}{ }^{e} \delta g_{d e}-2 E_{R}^{a c b d} \nabla_{d} \nabla_{c} \delta g_{a b} \\
& =\left(E_{R}^{c d e a} R_{c d e}{ }^{b}-2 \nabla_{c} \nabla_{d} E_{R}^{a c b d}\right) \delta g_{a b}+2 \nabla_{d}\left(\nabla_{c} E_{R}^{a d b c} \delta g_{a b}-E_{R}^{a c b d} \nabla_{c} \delta g_{a b}\right),
\end{aligned}
$$


For the third term of eq. (2.5), we have

$$
E_{F}^{a b} \delta F_{a b}=2 E_{F}^{a b} \nabla_{a} \delta A_{b}=-2 \nabla_{a} E_{F}^{a b} \delta A_{b}+2 \nabla_{d}\left(E_{F}^{d b} \delta A_{b}\right) .
$$

Summing the above results, we can get

$$
\delta \mathcal{L}=-\left(E_{R}^{c d e a} R_{c d e}^{b}+2 \nabla_{c} \nabla_{d} E_{R}^{a c b d}+E_{F}^{a c} F_{c}^{b}\right) \delta g_{a b}-2 \nabla_{a} E_{F}^{a b} \delta A_{b}+\nabla_{d} \delta v^{d}
$$

with

$$
\delta v^{d}=2 \nabla_{c} E_{R}^{a d b c} \delta g_{a b}-2 E_{R}^{a c b d} \nabla_{c} \delta g_{a b}+2 E_{F}^{d b} \delta A_{b} .
$$

Using

$$
\nabla_{d} \delta v^{d}=d \star \boldsymbol{\delta} \boldsymbol{v}
$$

we can further obtain

$$
\boldsymbol{\Theta}(\phi, \delta \phi)=\boldsymbol{\Theta}^{\text {grav }}(\phi, \delta g)+\boldsymbol{\Theta}^{\text {e.m. }}(\phi, \delta \boldsymbol{A}),
$$

in which

$$
\begin{aligned}
\Theta_{a_{2} \cdots a_{n}}^{\text {grav }}(\phi, \delta g) & =\boldsymbol{\epsilon}_{c a_{2} \cdots a_{n}}\left(2 \nabla_{d} E_{R}^{a c b d} \delta g_{a b}+2 E_{R}^{a b c d} \nabla_{b} \delta g_{a d}\right), \\
\Theta_{a_{2} \cdots a_{n}}^{\mathrm{e} . \mathrm{m} .}(\phi, \delta \boldsymbol{A}) & =2 \boldsymbol{\epsilon}_{a a_{2} \cdots a_{n}} E_{F}^{a b} \delta A_{b} .
\end{aligned}
$$

Moreover, we also have

$$
\boldsymbol{E}_{\phi} \delta \phi=-\boldsymbol{\epsilon}\left(\frac{1}{2} T^{a b} \delta g_{a b}+j^{a} \delta A_{a}\right)
$$

in which

$$
\begin{aligned}
T^{a b} & =2 E_{R}^{c d e(a} R_{c d e}{ }^{b)}+4 \nabla_{c} \nabla_{d} E_{R}^{(a|c| b) d}+2 E_{F}^{(a|c|} F^{b)}{ }_{c}-g^{a b} \mathcal{L}, \\
j^{b} & =2 \nabla_{a} E_{F}^{a b}
\end{aligned}
$$

can be regarded as the stress-energy tensor and electric current of the extra matter source.

Using the symplectic potential, the symplectic current $(n-1)$-form is defined by

$$
\boldsymbol{\omega}\left(\phi, \delta_{1} \phi, \delta_{2} \phi\right)=\delta_{2} \boldsymbol{\Theta}\left(\phi, \delta_{1} \phi\right)-\delta_{1} \boldsymbol{\Theta}\left(\phi, \delta_{2} \phi\right),
$$

in which $\delta_{1}$ and $\delta_{2}$ are the variations related to any two different one-parameter families. If the spacetime $M$ is global hyperbolic, denoting $C$ to the Cauchy surface, the symplectic form of this theory is defined as

$$
\Omega\left(\phi, \delta_{1} \phi, \delta_{2} \phi\right)=\int_{C} \boldsymbol{\omega}\left(\phi, \delta_{1} \phi, \delta_{2} \phi\right) .
$$

The Noether current $(n-1)$-form related to the vector field $\zeta^{a}$ is defined as

$$
\boldsymbol{J}_{\zeta}=\boldsymbol{\Theta}\left(\phi, \mathcal{L}_{\zeta} \phi\right)-\zeta \cdot \boldsymbol{L} .
$$

Using eq. (2.3), it is not hard to verify

$$
d \boldsymbol{J}_{\zeta}=-\boldsymbol{E}_{\phi} \mathcal{L}_{\zeta} \phi
$$

Therefore, if the dynamical field $\phi$ satisfy the on-shell condition $\boldsymbol{E}_{\phi}=0$, the Noether current is a closed form, i.e., $d \boldsymbol{J}_{\zeta}=0$, which implies there is a Noether charge $(n-2)$-form $\boldsymbol{Q}_{\zeta}$ such that $\boldsymbol{J}=d \boldsymbol{Q}$. Next, we prove the following lemma: 
Lemma 1. For the theory with Lagrangian (2.1), the Noether current $\boldsymbol{J}_{\zeta}$ can be divided as

$$
\boldsymbol{J}_{\zeta}=\boldsymbol{C}_{\zeta}+d \boldsymbol{Q}_{\zeta}
$$

in which

$$
\begin{aligned}
& \left(\boldsymbol{C}_{\zeta}\right)_{a_{2} \cdots a_{n}}=\boldsymbol{\epsilon}_{a a_{2} \cdots a_{n}}\left(\zeta^{b} T_{b}{ }^{a}+\zeta^{b} A_{b} j^{a}\right), \\
& \left(\boldsymbol{Q}_{\zeta}\right)_{a_{3} \cdots a_{n}}=\boldsymbol{\epsilon}_{a b a_{3} \cdots a_{n}}\left(E_{F}^{a b} A^{c} \zeta_{c}-2 \nabla_{d} E_{R}^{a b c d} \zeta_{c}-E_{R}^{a b c d} \nabla_{[c} \zeta_{d]}\right) .
\end{aligned}
$$

are the constraint $(n-1)$-form and Noether charge $(n-2)$-form of this theory separately. When the dynamical field $\phi$ satisfies the on-shell condition, we have $\boldsymbol{C}_{\zeta}=0$.

Proof. The Noether current $\boldsymbol{J}_{\zeta}$ can be written as

$$
\begin{aligned}
\boldsymbol{J}_{\zeta} & =\boldsymbol{\Theta}\left(\phi, \mathcal{L}_{\zeta} \phi\right)-\zeta \cdot \boldsymbol{L} \\
& =\star\left(\boldsymbol{v}_{\zeta}-\zeta \mathcal{L}\right),
\end{aligned}
$$

in which we have defined $\boldsymbol{v}_{\zeta}=\left.\delta \boldsymbol{v}\right|_{\delta \phi=\mathcal{L}_{\zeta} \phi}$. Based on eq. (2.14), the first term of above equation can be expressed as

$$
\begin{aligned}
v_{\zeta}^{c}= & 2 E_{R}^{a b c d} \nabla_{b}\left(\mathcal{L}_{\zeta} g_{a d}\right)+2 \mathcal{L}_{\zeta} g_{b d} W^{c b d}+E_{1}^{c} \mathcal{L}_{\zeta} \chi \\
& +E_{2}^{c b} \nabla_{b}\left(\mathcal{L}_{\zeta} \chi\right)-\nabla_{b} E_{2}^{b c} \mathcal{L}_{\zeta} \chi+2 E_{F}^{c b} \mathcal{L}_{\zeta} A_{b} .
\end{aligned}
$$

Using

$$
\begin{aligned}
\mathcal{L}_{\zeta} g_{a b} & =2 \nabla_{(a} \zeta_{b)}, \\
\mathcal{L}_{\zeta} A_{a} & =\nabla_{a}\left(\zeta^{b} A_{b}\right)+\zeta^{b} F_{b a},
\end{aligned}
$$

we have

$$
v_{\zeta}^{c}=4 E_{R}^{a b c d} \nabla_{b} \nabla_{(a} \zeta_{d)}+4 \nabla_{(b} \zeta_{d)} \nabla_{a} E_{R}^{a b c d}+2 E_{F}^{c b} \nabla_{b}\left(\zeta^{a} A_{a}\right)+2 E_{F}^{c b} \zeta^{a} F_{a b} .
$$

For the first term of above expression, we have

$$
\begin{aligned}
v_{12}^{c}= & 2 E_{R}^{a b c d} \nabla_{b} \nabla_{a} \zeta_{d}+2 E_{R}^{a b c d} \nabla_{b} \nabla_{d} \zeta_{a}+2 \nabla_{b} \zeta_{d} \nabla_{a} E_{R}^{a b c d}+2 \nabla_{d} \zeta_{b} \nabla_{a} E_{R}^{a b c d} \\
= & 2 E_{R}^{a b c d} \nabla_{[b} \nabla_{a]} \zeta_{d}+4 E_{R}^{a b c d} \nabla_{[b} \nabla_{d]} \zeta_{a}+2 E_{R}^{a b c d} \nabla_{d} \nabla_{b} \zeta_{a} \\
& +2 \nabla_{b} \zeta_{d} \nabla_{a} E_{R}^{a b c d}+2 \nabla_{d} \zeta_{b} \nabla_{a} E_{R}^{a b c d} \\
= & E_{R}^{a b d c} R_{a b d e} \zeta^{e}+2 E_{R}^{a b c d} R_{b d a e} \zeta^{e}+2 \nabla_{d}\left(E_{R}^{a b c d} \nabla_{b} \zeta_{a}\right) \\
& -2 \nabla_{b} \zeta_{a} \nabla_{d} E_{R}^{a b c d}+2 \nabla_{b} \zeta_{d} \nabla_{a} E_{R}^{a b c d}+2 \nabla_{d} \zeta_{b} \nabla_{a} E_{R}^{a b c d} \\
= & E_{R}^{a b d c} R_{a b d e} \zeta^{e}+2 E_{R}^{a b c d} R_{b d a e} \zeta^{e}+2 \nabla_{d}\left(E_{R}^{a b c d} \nabla_{b} \zeta_{a}\right)-2 \nabla_{b}\left(\zeta_{a} \nabla_{d} E_{R}^{a b c d}\right) \\
& +2 \nabla_{b}\left(\zeta_{d} \nabla_{a} E_{R}^{a b c d}\right)+2 \nabla_{d}\left(\zeta_{b} \nabla_{a} E_{R}^{a b c d}\right)+2 \zeta_{a} \nabla_{b} \nabla_{d} E_{R}^{a b c d} \\
& -2 \zeta_{d} \nabla_{b} \nabla_{a} E_{R}^{a b c d}-2 \zeta_{b} \nabla_{d} \nabla_{a} E_{R}^{a b c d} \\
= & E_{R}^{a b d c} R_{a b d e} \zeta^{e}+2 E_{R}^{a b c d} R_{b d a e} \zeta^{e}+2 \zeta_{d} \nabla_{a} \nabla_{b}\left(E_{R}^{d a c b}+E_{R}^{a b c d}-E_{R}^{b d c a}\right) \\
& +2 \nabla_{d}\left[E_{R}^{a b c d} \nabla_{b} \zeta_{a}+\zeta_{b} \nabla_{a}\left(E_{R}^{a b c d}-E_{R}^{d a c b}-E_{R}^{b d c a}\right)\right] .
\end{aligned}
$$

From the definition of $E_{R}^{a b c d}$, we can see that $E_{R}^{a b c d}$ has the same symmetries as $R_{a b c d}$. Therefore, $E_{R}^{a b c d}$ also satisfies the Bianchi identity $E_{R}^{[a b c] d}=0$, i.e.,

$$
E_{R}^{a b c d}+E_{R}^{b d c a}+E_{R}^{d a c b}=0 .
$$


Using the above identity, we have

$$
\begin{aligned}
v_{12}^{c}= & E_{R}^{a b d c} R_{a b d e} \zeta^{e}+2 E_{R}^{a b c d} R_{b d a e} \zeta^{e}-4 \zeta_{d} \nabla_{a} \nabla_{b} E_{R}^{b d c a} \\
& +2 \nabla_{d}\left[E_{R}^{a b c d} \nabla_{b} \zeta_{a}+2 \zeta_{b} \nabla_{a} E_{R}^{a b c d}\right] \\
= & E_{R}^{a b d c} R_{a b d e} \zeta^{e}+2 E_{R}^{a b c d} R_{b d a e} \zeta^{e}+4 \zeta_{d} \nabla_{(a} \nabla_{b)} E_{R}^{c a d b}+4 \zeta_{d} \nabla_{[a} \nabla_{b]} E_{R}^{c a d b} \\
& +2 \nabla_{d}\left[E_{R}^{a b c d} \nabla_{b} \zeta_{a}+2 \zeta_{b} \nabla_{a} E_{R}^{a b c d}\right] \\
= & E_{R}^{a b d c} R_{a b d e} \zeta^{e}+4 \zeta_{d} \nabla_{a} \nabla_{b} E_{R}^{(c|a| d) b}+2 E_{R}^{a b c d} R_{b d a e} \zeta^{e}-2 R_{a b e}{ }^{c} E_{R}^{e a d b} \zeta_{d} \\
& -2 R_{a b e}{ }^{d} E_{R}^{c a e b} \zeta_{d}+2 \nabla_{d}\left[E_{R}^{a b c d} \nabla_{b} \zeta_{a}+2 \zeta_{b} \nabla_{a} E_{R}^{a b c d}\right] .
\end{aligned}
$$

Considering the following results

$$
\begin{aligned}
& 2 E_{R}^{a b c d} R_{b d a e} \zeta^{e}=E_{R}^{a b c d} R_{b d a e} \zeta^{e}+E_{R}^{a b c d} R_{d a b e} \zeta^{e}=E_{R}^{a b d c} R_{a b d e} \zeta^{e}, \\
& 2 R_{a b e}{ }^{c} E_{R}^{e a d b} \zeta_{d}=R_{a b e}{ }^{c} E_{R}^{a b e d} \zeta_{d}, \quad 2 R_{a b e}{ }^{d} E_{R}^{c a e b} \zeta_{d}=-R_{b e a}{ }^{d} E_{R}^{b e a c} \zeta_{d},
\end{aligned}
$$

we can further obtain

$$
\begin{aligned}
v_{12}^{c}= & 3 E_{R}^{a b d c} R_{a b d e} \zeta^{e}-E_{R}^{a b d e} R_{a b d}^{c} \zeta_{e}+4 \zeta_{d} \nabla_{a} \nabla_{b} E_{R}^{(c|a| d) b} \\
& +2 \nabla_{d}\left(E_{R}^{a b c d} \nabla_{b} \zeta_{a}+2 \zeta_{b} \nabla_{a} E_{R}^{a b c d}\right) \\
= & 4 E_{R}^{a b d[c} R_{a b d}^{e]} \zeta_{e}+2 E_{R}^{a b d(c} R_{a b d}^{e)} \zeta_{e}+4 \zeta_{d} \nabla_{a} \nabla_{b} E_{R}^{(c|a| d) b} \\
& +2 \nabla_{d}\left(E_{R}^{a b c d} \nabla_{b} \zeta_{a}+2 \zeta_{b} \nabla_{a} E_{R}^{a b c d}\right) .
\end{aligned}
$$

For the third and forth terms of eq. (2.29), we have

$$
v_{34}^{c}=2 E_{F}^{c b} \nabla_{b}\left(\zeta^{a} A_{a}\right)+2 E_{F}^{c b} \zeta^{a} F_{a b}=2 \nabla_{d}\left(E_{F}^{c d} \zeta^{a} A_{a}\right)+j^{c} A_{a} \zeta^{a}+2 E_{F}^{c b} \zeta^{a} F_{a b} .
$$

Summing the above results, we have

$$
\begin{aligned}
v_{\zeta}^{c}= & 3 E_{R}^{a b d c} R_{a b d e} \zeta^{e}-E_{R}^{a b d e} R_{a b d}{ }^{c} \zeta_{e}+2 E_{F}^{c b} \zeta^{a} F_{a b}+4 \zeta_{d} \nabla_{a} \nabla_{b} E_{R}^{(c|a| d) b} \\
& +j^{c} A_{e} \zeta^{e}+2 \nabla_{d}\left(E_{R}^{a b c d} \nabla_{b} \zeta_{a}+2 \zeta_{b} \nabla_{a} E_{R}^{a b c d}+E_{F}^{c d} \zeta^{a} A_{a}\right) \\
= & \left(4 E_{R}^{a b d[c} R_{a b d}^{e]}+2 E_{F}^{b[c} F_{b}^{e]}\right) \zeta_{e}+\left(2 E_{R}^{a b d(c} R_{a b d}^{e)}+2 E_{F}^{b(c} F^{e)}{ }_{b}+4 \nabla_{a} \nabla_{b} E_{R}^{(c|a| e) b}\right) \zeta_{e} \\
& +j^{c} A_{e} \zeta^{e}+2 \nabla_{d}\left(E_{R}^{a b c d} \nabla_{b} \zeta_{a}+2 \zeta_{b} \nabla_{a} E_{R}^{a b c d}+E_{F}^{c d} \zeta^{a} A_{a}\right) .
\end{aligned}
$$

From eq. (2.10), we can see that the first term of the above expression vanishes. Together with the equation of motion (2.19), we can get

$$
v_{\zeta}^{c}-\zeta^{c} \mathcal{L}=\zeta^{e} T_{e}^{c}+\zeta^{e} A_{e} j^{c}+2 \nabla_{d}\left(E_{R}^{a b c d} \nabla_{b} \zeta_{a}+2 \zeta_{b} \nabla_{a} E_{R}^{a b c d}+E_{F}^{c d} \zeta^{a} A_{a}\right) .
$$

Therefore, we have

$$
\boldsymbol{J}_{\zeta}=\boldsymbol{C}_{\zeta}+d \boldsymbol{Q}_{\zeta}
$$

with

$$
\begin{aligned}
& \left(\boldsymbol{C}_{\zeta}\right)_{a_{2} \cdots a_{n}}=\boldsymbol{\epsilon}_{a a_{2} \cdots a_{n}}\left(\zeta^{b} T_{b}{ }^{a}+\zeta^{b} A_{b} j^{a}\right), \\
& \left(\boldsymbol{Q}_{\zeta}\right)_{a_{3} \cdots a_{n}}=\boldsymbol{\epsilon}_{a b a_{3} \cdots a_{n}}\left(E_{F}^{a b} A^{c} \zeta_{c}-2 \nabla_{d} E_{R}^{a b c d} \zeta_{c}-E_{R}^{a b c d} \nabla_{[c} \zeta_{d]}\right) .
\end{aligned}
$$

As we desired to show. 
Variation of Noether current $\boldsymbol{J}_{\zeta}$ from eq. (2.24), we can get

$$
\begin{aligned}
\bar{\delta} \boldsymbol{J}_{\zeta} & =\bar{\delta} \boldsymbol{\Theta}\left(\phi, \mathcal{L}_{\zeta} \phi\right)-\zeta \cdot \delta \boldsymbol{L} \\
& =\bar{\delta} \boldsymbol{\Theta}\left(\phi, \mathcal{L}_{\zeta} \phi\right)-\zeta \cdot \boldsymbol{E}_{\phi} \delta \phi-\zeta \cdot d \boldsymbol{\Theta}(\phi, \delta \phi) \\
& =\bar{\delta} \boldsymbol{\Theta}\left(\phi, \mathcal{L}_{\zeta} \phi\right)-\mathcal{L}_{\zeta} \boldsymbol{\Theta}(\phi, \delta \phi)+d[\zeta \cdot \boldsymbol{\Theta}(\phi, \delta \phi)]-\zeta \cdot \boldsymbol{E}_{\phi} \delta \phi \\
& =\boldsymbol{\omega}\left(\phi, \delta \phi, \mathcal{L}_{\zeta} \phi\right)+d[\zeta \cdot \boldsymbol{\Theta}(\phi, \delta \phi)]-\zeta \cdot \boldsymbol{E}_{\phi} \delta \phi,
\end{aligned}
$$

where we have introduce the notation $\bar{\delta}$ to denote the variation when the vector field $\zeta^{a}$ is fixed, i.e., we have

$$
\delta X_{\zeta}=\bar{\delta} X_{\zeta}+X_{\delta \zeta}
$$

for the quantity $X_{\zeta}$.

Moreover, using eq. (2.26), we have

$$
\bar{\delta} \boldsymbol{J}_{\zeta}=\bar{\delta} \boldsymbol{C}_{\zeta}+d \bar{\delta} \boldsymbol{Q}_{\zeta}
$$

Combining the above results, we can obtain the following identity

$$
d\left[\bar{\delta} \boldsymbol{Q}_{\zeta}-\zeta \cdot \boldsymbol{\Theta}(\phi, \delta \phi)\right]=\boldsymbol{\omega}\left(\phi, \delta \phi, \mathcal{L}_{\zeta} \phi\right)-\zeta \cdot \boldsymbol{E}_{\phi} \delta \phi-\bar{\delta} \boldsymbol{C}_{\zeta}
$$

In the following, we consider a one-parameter family $\phi(\lambda)$ in which any $\phi(\lambda)$ satisfy the on-shell condition, i.e., we have $\boldsymbol{C}(\lambda)=\boldsymbol{E}_{\phi}(\lambda)=0$ and $\delta \boldsymbol{C}=\boldsymbol{E}_{\phi}=0$. Then, the variational identity becomes

$$
\boldsymbol{\omega}\left(\phi, \delta \phi, \mathcal{L}_{\zeta} \phi\right)=d\left[\bar{\delta} \boldsymbol{Q}_{\zeta}-\zeta \cdot \boldsymbol{\Theta}(\phi, \delta \phi)\right]
$$

Consider the asymptotically flat stationary-axisymmetric spacetime satisfying the asymptotic condition of "Case I" in ref. [18]. Let $\zeta^{a}$ be a vector field related to the symmetry at asymptotically infinity. Then, there exists a conserved quantity $H_{\zeta}$ related to this vector field. If we assume $\phi(\lambda)$ satisfies the on-shell condition, $\delta H_{\zeta}$ can be expressed as $[8,18]$

$$
\delta H_{\zeta}=\int_{\infty}\left(\bar{\delta} \boldsymbol{Q}_{\zeta}-\zeta \cdot \boldsymbol{\Theta}\right)
$$

in which " $\infty$ " denotes a $(n-2)$-sphere at asymptotically infinity. When $\zeta^{a}$ is chosen as the vector field $t^{a}$ related to the asymptotic time translation or $\varphi^{a}$ related to the rotation, the canonical mass and angular momentum can be defined by [8]

$$
\delta M=\int_{\infty}\left(\bar{\delta} \boldsymbol{Q}_{t}-t \cdot \boldsymbol{\Theta}\right), \quad \delta J=\int_{\infty}\left(\bar{\delta} \boldsymbol{Q}_{\varphi}-\varphi \cdot \boldsymbol{\Theta}\right)
$$

Using the equation of motion (2.19), the electric charge of the spacetime is defined by

$$
Q=-\int_{\infty} \epsilon_{a b a_{3} \cdots a_{n}} E_{F}^{a b}
$$




\section{The first law of the stationary-axisymmetric black holes}

In this section, we would like to derive the first law of black holes in the gravitational electromagnetic system with Lagrangian (2.1). Let $\left(M, g_{a b}\right)$ is an asymptotically flat stationaryaxisymmetric spacetime satisfying the asymptotic condition of "Case I" in ref. [18], and there is a bifurcated Killing horizon $\mathcal{H}$ with a compact bifurcated surface $\mathcal{B}$. Assume that the metric $g_{a b}$ and electromagnetic strength $F_{a b}$ is smooth near the horizon as well as the bifurcation surface. The generated Killing vector field of the Killing horizon can be expressed as

$$
\xi^{a}=t^{a}+\Omega_{H} \varphi^{a},
$$

in which we have denoted $\Omega_{H} \varphi^{a}=\Omega_{H}^{(\mu)} \varphi_{(\mu)}^{a}$. Here $t^{a}$ and $\varphi_{(\mu)}^{a}$ are the Killing vector fields related to the time transition and axial symmetries of the spacetime, $\Omega_{H}^{(\mu)}$ is the velocity of the black hole horizon $\mathcal{H}$. Considering the gauge covariance of the electromagnetic field $A_{a}$, we can impose the conditions

$$
\left.A_{a} \xi^{a}\right|_{\infty}=0 \quad \text { and } \quad \mathcal{L}_{\xi} A_{a}=0
$$

such that $A_{a}$ is a Killing vector field in the spacetime. This condition always can be imposed by a gauge transformation $A_{a} \rightarrow A_{a}^{\prime}=A_{a}-\nabla_{a} \chi$ with $\chi$ satisfying $\left.\xi^{a} \nabla_{a} \chi\right|_{\infty}=\left.A_{a} \xi^{a}\right|_{\infty}$ and $\nabla_{a}\left(\xi^{b} \nabla_{b} \chi\right)=\mathcal{L}_{\xi} A_{a}$

In the following, we consider a one-parameter family $\phi(\lambda)$, any element in which is a stationary-axisymmetric black hole as described above. Considering the diffeomorphism invariance of the theory, we can choose a gauge such that $\xi^{a}$ and Killing horizon $\mathcal{H}$ (including the bifurcation surface $\mathcal{B}$ ) is independent on $\lambda$, i.e., they are fixed under the variation. Replacing $\zeta^{a}$ by $\xi^{a}$ and considering the symmetries

$$
\mathcal{L}_{\xi} g_{a b}(\lambda)=0, \quad \mathcal{L}_{\xi} \boldsymbol{A}(\lambda)=0
$$

the variational identity (2.43) implies

$$
d\left[\bar{\delta} \boldsymbol{Q}_{\xi}-\xi \cdot \boldsymbol{\Theta}(\phi, \delta \phi)\right]=0 .
$$

Choose $\Sigma$ to a hypersurface connecting the sphere $S_{\infty}$ at infinity and a cross-section $S$ on the future Killing horizon. Integration of eq. (3.4) on $\Sigma$, using the Stokes theorem, we can further obtain

$$
\int_{\infty}\left[\bar{\delta} \boldsymbol{Q}_{\xi}-\xi \cdot \boldsymbol{\Theta}(\phi, \delta \phi)\right]=\int_{S}\left[\bar{\delta} \boldsymbol{Q}_{\xi}-\xi \cdot \boldsymbol{\Theta}(\phi, \delta \phi)\right] .
$$

For the left side of the above expression, using the definition of the mass and angular motion (2.45), we have

$$
\begin{aligned}
\int_{\infty}\left[\bar{\delta} \boldsymbol{Q}_{\xi}-\xi \cdot \boldsymbol{\Theta}(\phi, \delta \phi)\right] & =\int_{\infty}\left[\bar{\delta} \boldsymbol{Q}_{\xi}-t \cdot \boldsymbol{\Theta}(\phi, \delta \phi)\right] \\
& =\int_{\infty}\left[\bar{\delta} \boldsymbol{Q}_{t}-t \cdot \boldsymbol{\Theta}(\phi, \delta \phi)\right]+\int_{\infty} \bar{\delta} \boldsymbol{Q}_{\Omega_{H} \varphi} \\
& =\delta M-\Omega_{H} \delta J
\end{aligned}
$$


For the right side of eq. (3.5), considering the gauge choice $\delta \xi^{a}=0$, we can replace $\bar{\delta}$ by $\delta$. Then, we have

$$
\begin{aligned}
\int_{S}\left[\bar{\delta} \boldsymbol{Q}_{\xi}-\xi \cdot \boldsymbol{\Theta}(\phi, \delta \phi)\right]= & \delta \int_{S} \boldsymbol{\epsilon}_{a b a_{3} \cdots a_{n}}\left[E_{F}^{a b} A^{c} \xi_{c}-2 \xi_{c} \nabla_{d} E_{R}^{a b c d}-E_{R}^{a b c d} \nabla_{[c} \xi_{d]}\right] \\
& -\int_{S} \xi \cdot\left[\boldsymbol{\Theta}^{\mathrm{e} . \mathrm{m}}(\phi, \delta \boldsymbol{A})+\boldsymbol{\Theta}^{\mathrm{grav}}(\phi, \delta g)\right] .
\end{aligned}
$$

Since we assume that $R_{a b c d}, g_{a b}$ and $F_{a b}$ is smooth near the Killing horizon (including the bifurcation surface $\mathcal{B}), E_{R}^{a b c d}$ and $E_{F}^{a b}$ would also be the smooth tensor near the horizon. Before deriving the first law, we first prove the following Lemma:

Lemma 2. For a stationary black hole with bifurcated Killing horizon $\mathcal{H}$. Let $\xi^{a}$ be a Killing vector field generated the future Killing horizon, $S$ be a cross-section of future horizon, and $s^{a}$ is another null vector field on $\mathcal{H}$ satisfying

$$
s^{a} \xi_{a}=1, \quad s^{a} s_{a}=0, \quad w_{i}^{a} s_{a}=0,
$$

in which $w_{i}^{a}$ is the tangent vector on the cross section $S$. Denote $z_{i}^{a}=\left\{\xi^{a}, s^{a}, w_{j}^{a}\right\}$ to the basis on the cross section. Then, for any tensor field $X_{a_{1} \cdots a_{k}}$ which is smooth on the horizon (including bifurcation surface) and satisfies $\mathcal{L}_{\xi} X=X_{a_{1} \cdots a_{k}}$, if $X_{a_{1} \cdots a_{k}} z_{1}^{a} \cdots z_{k}^{a}$ is not zero, the number of $\xi^{a}$ must not be greater than the number of $s^{a}$.

Proof. Consider a foliation of the horizon $\mathcal{H}$ which is obtained from the cross-section $S$ by the diffeomorphism generated by the Killing vector field $\xi^{a}$. The vector field $z_{i}^{a}$ is also generated by this diffeomorphism, i.e., we have

$$
\mathcal{L}_{\xi} z_{i}^{a}=0
$$

For the slice (cross section) $S$ is not the bifurcation surface $\mathcal{B}, z_{i}^{a}$ would be a finite vector field, i.e., contraction of any finite tensors is finite. Since $X_{a_{1} \cdots a_{k}}$ is smooth on the Killing horizon $\mathcal{H}, X_{a_{1} \cdots a_{k}} z_{1}^{a} \cdots z_{k}^{a}$ would be finite on any cross section $S$.

When $S$ approach the bifurcation surface $\mathcal{B}$, we have $\xi^{a} \rightarrow 0$. However, note $s^{a} \xi_{a}$ is finite, $s^{a}$ must be divergent when $S \rightarrow \mathcal{B}$. To show the divergence, we choose another two finite null vector field $k^{a}$ and $l^{a}$ on the cross-section $S$ near the bifurcation surface, which satisfies

$$
\begin{aligned}
& k^{a} l_{a}=-1, \quad k_{a} k^{a}=0, \quad l_{a} l^{a}=0, \\
& k^{a}=C \xi^{a}, \quad l^{a}=C^{-1} s^{a},
\end{aligned}
$$

in which $C$ is a scalar field on the cross section $S$. Since $k^{a}$ and $l^{a}$ are finite vectors on the bifurcation surface, we have $C \rightarrow \infty$ when $S \rightarrow \mathcal{B}$. Denote $\bar{z}_{i}^{a}=\left\{k^{a}, l^{a}, w_{i}^{a}\right\}$. Since we assume that $X_{a_{1} \cdots a_{k}}$ is smooth near the bifurcation surface $\mathcal{B}, X_{a_{1} \cdots a_{k}} \bar{z}_{1}^{a} \cdots \bar{z}_{k}^{a}$ will be finite on $\mathcal{B}$. Since

$$
\mathcal{L}_{\xi} X_{a_{1} \cdots a_{k}}=0
$$

we have

$$
\mathcal{L}_{\xi}\left(X_{a_{1} \cdots a_{k}} z_{1}^{a} \cdots z_{k}^{a}\right)=0
$$


which implies that $X_{a_{1} \cdots a_{k}} z_{1}^{a} \cdots z_{k}^{a}$ is invariance along the Killing vector $\xi^{a}$. When the cross section $S$ is not the bifurcation surface $\mathcal{B}, z^{a}$ would be a finite vector. Then, we have $X_{a_{1} \cdots a_{k}} z_{1}^{a} \cdots z_{k}^{a}$ is finite on whole future horizon $\mathcal{H}$. From eq. (3.10), we have

$$
X_{a_{1} \cdots a_{k}} \bar{z}_{1}^{a} \cdots \bar{z}_{k}^{a}=C^{m-n} X_{a_{1} \cdots a_{k}} z_{1}^{a} \cdots z_{k}^{a},
$$

in which $m$ is the number of $\xi^{a}$ and $n$ is the number of $s^{a}$. Since $X_{a_{1} \cdots a_{k}}$ is a smooth tensor near the bifurcation surface, $X_{a_{1} \cdots a_{k}} \bar{z}_{1}^{a} \cdots \bar{z}_{k}^{a}$ should be finite when $C$ approaches $\mathcal{B}$. However, from eq. (3.13), we can see that when $m>n$, if $X_{a_{1} \cdots a_{k}} z_{1}^{a} \cdots z_{k}^{a}$ is finite, $X_{a_{1} \cdots a_{k}} \bar{z}_{1}^{a} \cdots \bar{z}_{k}^{a}$ would be divergent, which is in contradiction with the assumption that $X_{a_{1} \cdots a_{k}} \bar{z}_{1}^{a}$ is smooth near the bifurcation surface. Therefore, when $m>n$, we must have

$$
X_{a_{1} \cdots a_{k}} z_{1}^{a} \cdots z_{k}^{a}=0
$$

As we desired to show.

Since we assume that $E_{R}^{a b c d}$ is smooth near horizon, $\nabla_{a} E_{R}^{a b c d}$ is also smooth near the horizon (including bifurcation surface). Using lemma 2, we have

$$
\boldsymbol{\epsilon}_{a b a_{3} \cdots a_{n}} \xi_{c} \nabla_{d} E_{R}^{a b c d}=2 \tilde{\boldsymbol{\epsilon}}_{a_{3} \cdots a_{n}} s_{a} \xi_{b} \xi_{c} \nabla_{d} E_{R}^{a b c d}=0
$$

on the horizon $\mathcal{H}$. Then, eq. (3.7) can reduce to

$$
\begin{aligned}
& \int_{S}\left[\bar{\delta} \boldsymbol{Q}_{\xi}-\xi \cdot \boldsymbol{\Theta}(\phi, \delta \phi)\right] \\
= & \delta \int_{S} \boldsymbol{\epsilon}_{a b a_{3} \cdots a_{n}}\left[E_{F}^{a b} A^{c} \xi_{c}-E_{R}^{a b c d} \nabla_{[c} \xi_{d]}\right]-\int_{S} \xi \cdot\left[\boldsymbol{\Theta}^{\mathrm{e} . \mathrm{m}}(\phi, \delta \boldsymbol{A})+\boldsymbol{\Theta}^{\mathrm{grav}}(\phi, \delta g)\right] .
\end{aligned}
$$

For the first term of the left side in the above equation, we first prove that $A_{a} \xi^{a}$ is a constant on the horizon. Considering the Killing condition $\mathcal{L}_{\xi} A_{a}=0$, we can further obtain

$$
\nabla_{b}\left(\xi^{a} A_{a}\right)=-\xi^{a} F_{a b} .
$$

Using lemma 2 and the assumption $F_{a b}$ is smooth on the horizon (including bifurcation surface), we have $F_{a b} \xi^{a} \xi^{a}=F_{a b} \xi^{a} w_{i}^{b}=0$ on $\mathcal{H}$, which implies

$$
\xi^{b} \nabla_{b}\left(\xi^{a} A_{a}\right)=w_{i}^{b} \nabla_{b}\left(\xi^{a} A_{a}\right)=0
$$

on $\mathcal{H}$. Therefore, $\Phi_{H}=\left.A_{a} \xi^{a}\right|_{\infty}-\left.A_{a} \xi^{a}\right|_{\mathcal{H}}=-\left.A_{a} \xi^{a}\right|_{\mathcal{H}}$ is a constant on horizon. Then, the first term of the left side becomes

$$
\int_{S} \boldsymbol{\epsilon}_{a b a_{3} \cdots a_{n}} E_{F}^{a b} A^{c} \xi_{c}=-\Phi_{H} \int_{\mathcal{B}} \epsilon_{a b a_{3} \cdots a_{n}} E_{F}^{a b}
$$

Using the on-shell condition $\nabla_{a} E_{F}^{a b}=0$, it is not hard to get

$$
-\int_{\mathcal{B}} \epsilon_{a b a_{3} \cdots a_{n}} E_{F}^{a b}=-\int_{\infty} \epsilon_{a b a_{3} \cdots a_{n}} E_{F}^{a b}=Q .
$$

Therefore, we have

$$
\delta \int_{S} \epsilon_{a b a_{3} \cdots a_{n}} E_{F}^{a b} A^{c} \xi_{c}=\delta\left(\Phi_{H} Q\right)
$$


For the third term of eq. (3.16), we have

$$
\begin{aligned}
\int_{S} \xi \cdot \Theta^{\text {e.m. }}(\phi, \delta \boldsymbol{A}) & =2 \int_{S} \xi^{c} \boldsymbol{\epsilon}_{a c a_{3} \cdots a_{n}} E_{F}^{a b} \delta A_{b} \\
& =2 \int_{S} \tilde{\boldsymbol{\epsilon}} \xi^{c} \hat{\boldsymbol{\epsilon}}_{a c} E_{F}^{a b} \delta A_{b} .
\end{aligned}
$$

Using $\hat{\boldsymbol{\epsilon}}=s \wedge \xi=d v \wedge d r$, we can obtain

$$
\begin{aligned}
\int_{S} \xi \cdot \boldsymbol{\Theta}^{\mathrm{e} . \mathrm{m} \cdot}(\phi, \delta \boldsymbol{A}) & =2 \int_{S} \tilde{\boldsymbol{\epsilon}} \xi_{a} E_{F}^{a b} \delta A_{b}=2 \int_{S} \tilde{\boldsymbol{\epsilon}} \xi_{a} E_{F}^{a b} s_{b} \xi^{c} \delta A_{c} \\
& =\int_{S} \tilde{\boldsymbol{\epsilon}} \hat{\boldsymbol{\epsilon}}_{a b} E_{F}^{a b} \xi^{c} \delta A_{c}=Q \delta \Phi_{H} .
\end{aligned}
$$

In the following, we are going to evaluate the gravitational part. Since we choose the gauge such that $\xi^{a}$ and $\mathcal{H}$ is fixed in the variation, we can use the Gaussian null coordinates $\left\{v, r, \theta^{1}, \cdots, \theta^{n-2}\right\}$ to calculate these quantities. The line element of the spacetime in this coordinate can be expressed as [19]

$$
d s^{2}=2\left(d r-r \alpha d v-r \beta_{i} d \theta^{i}\right) d v+\gamma_{i j} d \theta^{i} d \theta^{j},
$$

in which $\alpha, \beta_{i}$ and $\gamma_{i j}$ are the function of $r$ and $\theta^{i}$. The horizon $\mathcal{H}$ is determined by $r=0$. The Killing vector field generated the horizon is

$$
\xi^{a}=\left(\frac{\partial}{\partial v}\right)^{a}, \quad s^{a}=\left(\frac{\partial}{\partial r}\right)^{a} .
$$

Note that the gauge choice which fixes the Gaussian null coordinates is the same as the gauge choice to fix $\xi^{a}$ and $\mathcal{H}$. In this gauge choice, only $\alpha, \beta_{i}$ and $\gamma_{i j}$ are dependent on the parameter $\lambda$. Based on the above coordinates, on the horizon $r=0$, we have

$$
\nabla_{a} \xi_{b}=\kappa \hat{\boldsymbol{\epsilon}}_{a b}-\beta_{i} \xi_{[a}\left(d \theta^{i}\right)_{b]},
$$

in which $\kappa=\alpha(0)$ is the surface gravity of the Killing horizon $\mathcal{H}$. For the Einstein gravity, the constancy of the surface gravity (black hole zeroth law) has been proven using only the dominant energy condition of the matter field [20]. Recently, the proof has been extended to Lanczos-Lovelock theory with the additional assumption that the theory has a smooth limit to general relativity [21]. However, there is no general proof of the zero law for the theory in any diffeomorphism covariant theories. Without the gravitational equations, the zeroth law can also be checked if the exterior derivative of the twist of the horizon Killing field vanishes on the horizon [22]. In this case, the result can be applied to any gravitational theory. As a corollary of this, it implies that $\kappa$ is constant on the horizon for all static black holes or any stationary-axisymmetric black hole with the ' $t-\phi$ ' reflection isometry $[22,23]$. In this paper, we only consider the cases where the zero law is satisfied.

For the second term of eq. (3.16), we have

$$
\begin{aligned}
\int_{S} \boldsymbol{\epsilon}_{a b a_{3} \cdots a_{n}} E_{R}^{a b c d} \nabla_{[c} \xi_{d]} & =\int_{S} \tilde{\boldsymbol{\epsilon}} \hat{\boldsymbol{\epsilon}}_{a b} E_{R}^{a b c d} \nabla_{[c} \xi_{d]} \\
& =\kappa \int_{S} \tilde{\boldsymbol{\epsilon}} \hat{\boldsymbol{\epsilon}}_{a b} \hat{\boldsymbol{\epsilon}}_{c d} E_{R}^{a b c d}+\int_{S} \tilde{\boldsymbol{\epsilon}} \beta_{i} \hat{\boldsymbol{\epsilon}}_{a b} \xi_{c}\left(d \theta^{i}\right)_{d} E_{R}^{a b c d} .
\end{aligned}
$$


Using lemma 2 and considering that $\left(d \theta^{i}\right)^{d}$ is a tangent vector of $S$, the second term of the above expression vanishes. Thus we have

$$
\int_{S} \boldsymbol{\epsilon}_{a b a_{3} \cdots a_{n}} E_{R}^{a b c d} \nabla_{[c} \xi_{d]}=\kappa \int_{S} \tilde{\boldsymbol{\epsilon}} \hat{\boldsymbol{\epsilon}}_{a b} \hat{\boldsymbol{\epsilon}}_{c d} E_{R}^{a b c d}=-\frac{\kappa S}{2 \pi} .
$$

Therefore, eq. (3.16) reduces to

$$
-\delta \int_{S} \boldsymbol{\epsilon}_{a b a_{3} \cdots a_{n}} E_{R}^{a b c d} \nabla_{[c} \xi_{d]}=\frac{1}{2 \pi} \delta(\kappa S) .
$$

For the last term of eq. (3.16), we have

$$
\begin{aligned}
\int_{S} \xi \cdot \Theta^{\operatorname{grav}}(\phi, \delta g) & =2 \int_{S} \xi^{e} \boldsymbol{\epsilon}_{c e \cdots a_{n}}\left(\nabla_{d} E_{R}^{a c b d} \delta g_{a b}+E_{R}^{a b c d} \nabla_{b} \delta g_{a d}\right) \\
& =-2 \int_{S} \tilde{\boldsymbol{\epsilon}}\left(\xi_{c} \nabla_{d} E_{R}^{a c b d} \delta g_{a b}+\xi_{c} E_{R}^{a b c d} \nabla_{b} \delta g_{a d}\right) .
\end{aligned}
$$

For the line element (3.24) in the Gaussian null coordinates, straightforward calculation gives $\delta g_{a b}=\delta \gamma_{a b}$, in which $\gamma_{a b}=\gamma_{i j}\left(d \theta^{i}\right)_{a}\left(d \theta^{j}\right)_{b}$. Based on lemma 2, it is not hard to see that the first term of eq. (3.30) vanishes. For the last term of eq. (3.30), we have

$$
\xi_{c} E_{R}^{a b c d} \nabla_{b} \delta g_{a d}=-E_{R}^{\mu \sigma \rho r} \delta g_{\mu \rho ; \sigma} .
$$

Using the line element (3.24), lemma 2 can be presented as: if $E_{R}^{\mu \sigma \rho \tau}$ is not zero on horizon, the number of " $v$ " in the index must be larger than number of " $r$ ". Therefore, the nonvanishing contributions in eq. (3.31) from $\delta g_{\mu \rho ; \sigma}$ only comes from the term in which the number of " $v$ " larger that number of " $r$ ". Using the line element, it is not hard to find that the only nonvanishing component of $\delta g_{\mu \rho ; \sigma}$ is

$$
\delta g_{v v ; r}=-2 \delta \alpha=-2 \delta \kappa .
$$

Therefore, we have

$$
\int_{S} \xi \cdot \Theta^{\operatorname{grav}}(\phi, \delta g)=-4 \delta \kappa \int_{S} \tilde{\boldsymbol{\epsilon}} E_{R}^{v r v r}=\frac{1}{2 \pi} S \delta \kappa .
$$

Summing the above results, we have

$$
\delta M=\frac{\kappa}{2 \pi} \delta S+\Omega_{H}^{(\mu)} \delta J_{(\mu)}+\Phi_{H} \delta Q .
$$

Therefore, we derived the first law of black holes in the gravitational electromagnetic system and the expression is the same as the Einstein-Maxwell theory.

\section{Conclusion}

The first law of black hole in a diffeomorphism covariant theory is generally derived by Iyer and Wald [6]. However, their results bases on the requirement that all dynamical fields be smooth near the future Killing horizon as well as the bifurcation surface, and consequently their discussion cannot be simply extended to the cases with gauge symmetry. In this 
paper, we extended their discussion into the gravitational electromagnetic system without the requirement that the vector potential $\boldsymbol{A}$ is smooth near the horizon since it is not a real physical quantity.

Firstly, we calculated the Noether charge and variational identity of the gravitational electromagnetic theory with the Lagrangian $\mathcal{L}\left(g_{a b}, R_{a b c d}, F_{a b}\right)$. Then, using these results, we derived the thermodynamic first law of the asymptotically flat stationary-axisymmetric black holes. In contrast to the earlier discussion by Iyer and Wald, we only require that the electromagnetic strength $F_{a b}$ and metric $g_{a b}$ be smooth near the future horizon (including the bifurcation surface), without making any constraints for the vector potential $\boldsymbol{A}$. Under the above conditions, we obtain the first law of thermodynamics for the mass, angular momentum, and charge variation of the black hole. The result is the same as the expression of the first law in Einstein-Maxwell theory. Our investigation shows that the first law of black hole thermodynamics is also universal in an effective theory that takes into account the quantum corrections in Einstein-Maxwell theory, and Wald entropy is still the best choice to describe the entropy of a steady black hole.

\section{Acknowledgments}

We acknowledge financial supports from the National Natural Science Foundation of China (Grants No. 11775022, 11873044 and 12005080).

Open Access. This article is distributed under the terms of the Creative Commons Attribution License (CC-BY 4.0), which permits any use, distribution and reproduction in any medium, provided the original author(s) and source are credited.

\section{References}

[1] J.D. Bekenstein, Black holes and the second law, Lett. Nuovo Cim. 4 (1972) 737 [InSPIRE].

[2] J.M. Bardeen, B. Carter and S.W. Hawking, The Four laws of black hole mechanics, Commun. Math. Phys. 31 (1973) 161 [InSPIRE].

[3] J.D. Bekenstein, Generalized second law of thermodynamics in black hole physics, Phys. Rev. D 9 (1974) 3292 [INSPIRE].

[4] S.W. Hawking, Particle Creation by Black Holes, Commun. Math. Phys. 43 (1975) 199 [Erratum ibid. 46 (1976) 206] [INSPIRE].

[5] D. Sudarsky and R.M. Wald, Extrema of mass, stationarity, and staticity, and solutions to the Einstein Yang-Mills equations, Phys. Rev. D 46 (1992) 1453 [InSPIRE].

[6] V. Iyer and R.M. Wald, Some properties of Noether charge and a proposal for dynamical black hole entropy, Phys. Rev. D 50 (1994) 846 [gr-qc/9403028] [INSPIRE].

[7] R.M. Wald, Black hole entropy is the Noether charge, Phys. Rev. D 48 (1993) R3427 [gr-qc/9307038] [INSPIRE].

[8] S. Gao, The First law of black hole mechanics in Einstein-Maxwell and Einstein-Yang-Mills theories, Phys. Rev. D 68 (2003) 044016 [gr-qc/0304094] [INSPIRE]. 
[9] D.A. Rasheed, Nonlinear electrodynamics: Zeroth and first laws of black hole mechanics, hep-th/9702087 [INSPIRE].

[10] P.A. Cano and A. Murcia, Electromagnetic Quasitopological Gravities, JHEP 10 (2020) 125 [arXiv: 2007.04331] [INSPIRE].

[11] T. Jacobson and A. Mohd, Black hole entropy and Lorentz-diffeomorphism Noether charge, Phys. Rev. D 92 (2015) 124010 [arXiv:1507.01054] [INSPIRE].

[12] K. Prabhu, The First Law of Black Hole Mechanics for Fields with Internal Gauge Freedom, Class. Quant. Grav. 34 (2017) 035011 [arXiv:1511.00388] [INSPIRE].

[13] Z. Elgood, P. Meessen and T. Ortín, The first law of black hole mechanics in the Einstein-Maxwell theory revisited, JHEP 09 (2020) 026 [arXiv:2006.02792] [INSPIRE].

[14] B. Zwiebach, Curvature Squared Terms and String Theories, Phys. Lett. B 156 (1985) 315 [INSPIRE].

[15] D.J. Gross and E. Witten, Superstring Modifications of Einstein's Equations, Nucl. Phys. B 277 (1986) 1 [INSPIRE].

[16] A. Sen, Black Hole Entropy Function, Attractors and Precision Counting of Microstates, Gen. Rel. Grav. 40 (2008) 2249 [arXiv: 0708.1270] [INSPIRE].

[17] A. Dabholkar and S. Nampuri, Quantum black holes, Lect. Notes Phys. 851 (2012) 165 [arXiv:1208.4814] [INSPIRE].

[18] R.M. Wald and A. Zoupas, A General definition of 'conserved quantities' in general relativity and other theories of gravity, Phys. Rev. D 61 (2000) 084027 [gr-qc/9911095] [INSPIRE].

[19] S. Hollands, A. Ishibashi and R.M. Wald, A Higher dimensional stationary rotating black hole must be axisymmetric, Commun. Math. Phys. 271 (2007) 699 [gr-qc/0605106] [INSPIRE].

[20] S.W. Hawking, Black holes in general relativity, Commun. Math. Phys. 25 (1972) 152 [INSPIRE].

[21] R. Ghosh and S. Sarkar, Black Hole Zeroth Law in Higher Curvature Gravity, Phys. Rev. D 102 (2020) 101503 [arXiv: 2009.01543] [INSPIRE].

[22] I. Racz and R.M. Wald, Global extensions of space-times describing asymptotic final states of black holes, Class. Quant. Grav. 13 (1996) 539 [gr-qc/9507055] [INSPIRE].

[23] R.M. Wald, General Relativity, University of Chicago Press, Chicago U.S.A. (1984) [DOI]. 